\title{
On co-channel and adjacent channel interference mitigation in cognitive radio networks is
}

\author{
Donglin Hu, Shiwen Mao* \\ Department of Electrical and Computer Engineering, Auburn University, Auburn, AL 36849-5201, USA
}

\section{A R T I C L E I N F O}

\section{Article history:}

Received 19 December 2011

Received in revised form 17 February 2013

Accepted 24 February 2013

Available online 15 March 2013

\section{Keywords:}

Adjacent channel interference

Cognitive radio networks

Cross-layer optimization

Distributed algorithm

Dynamic spectrum access

Interference mitigation

Spectrum sensing

\begin{abstract}
A B S T R A C T
Cognitive radio (CR) is an emerging wireless communications paradigm of sharing spectrum among licensed (or, primary) and unlicensed (or, CR) users. In CR networks, interference mitigation is crucial not only for primary user protection, but also for the quality of service of $C R$ user themselves. In this paper, we consider the problem of interference mitigation via channel assignment and power allocation for CR users. A cross-layer optimization framework for minimizing both co-channel and adjacent channel interference is developed; the latter has been shown to have considerable impact in practical systems. Cooperative spectrum sensing, opportunistic spectrum access, channel assignment, and power allocation are considered in the problem formulation. We propose a reformulation-linearization technique (RLT) based centralized algorithm, as well as a distributed greedy algorithm that uses local information for near-optimal solutions. Both algorithms are evaluated with simulations and are shown quite effective for mitigating both types of interference and achieving high CR network capacity.
\end{abstract}

(c) 2013 Elsevier B.V. All rights reserved.

\section{Introduction}

A cognitive radio (CR) is a frequency-agile wireless communication device that can sense the radio environment and dynamically reconfigure its radio parameter and behavior to adapt to changes in the radio environment. $C R$ represents a paradigm change in spectrum regulation and sharing. Its high potential has attracted substantial interest from industry, academia, and policy makers. Considerable $C R$ research has been focused on developing effective spectrum sensing and access techniques, two core components of a CR system (e.g., see $[2,3]$ ). FCC has recently issued rules and guidelines for unlicensed operations in the TV bands that require a combination of

\footnotetext{
This paper was presented in part at IEEE MILCOM 2011, Baltimore, MD, USA, November 7-10, 2011 [1].

* Corresponding author.

E-mail addresses: dzh0003@tigermail.auburn.edu (D. Hu), smao@ieee.org (S. Mao).
}

device geo-location and database lookup for channel access [4].

Although the basic concept of CR is intuitive, there exist numerous challenging problems to be solved to fully harvest its potential. To support many bandwidth-intensive applications in CR networks, it is desirable to achieve high network throughput under the constraint of limited interference to primary users. Due to the use of open space as transmission medium, wireless network capacity is usually constrained by interference. A CR user's transmission will generate interference not only to the neighboring primary users, but also to other CR users sharing the same or adjacent channels. Therefore, interference mitigation is crucial not only for primary user protection, but also for the quality of service of CR user themselves. Effective interference mitigation techniques are indispensable to realize the high potential of CRs.

Generally, there are two types of interference that should be considered in such multi-channel environment. The first type, co-channel interference (CCI), is due to the coexisting transmitters occupying the same band as the 
victim receiver. A widely used approach for $\mathrm{CCI}$ mitigation is to assign different channels to the interfering transmitters near the victim receiver [5-7]. The second type is adjacent channel interference $(\mathrm{ACI})$, which is in the form of power leakage from adjacent channels. $\mathrm{ACI}$ is mainly due to imperfect design of transmit filters and amplifiers. The harmful impact of ACI on network throughput was demonstrated in a recent work [8]. ACI can also be mitigated by appropriate channel allocation and power control. Both types of interference should be considered in the design of $\mathrm{CR}$ network protocols.

In this paper, we consider a $C R$ network consisting of multiple $C R$ transmitter receiver pairs. The primary network comprises a base station sending data to primary users using a set of licensed channels. The CR nodes collaboratively sense the licensed channels and exploit spectrum opportunities for data transmission. We investigate the problem of maximizing the $\mathrm{CR}$ network throughput while bounding the interference to primary users. We incorporate several important components such as cooperative spectrum sensing, spectrum sensing errors, and opportunistic spectrum access into the cross-layer optimization framework. In the problem formulation, we specifically consider mitigating CCI among CR users and ACI for both $\mathrm{CR}$ and primary users, through optimized channel assignment and transmit power control for CR users.

The formulated problem is a Mixed Integer Nonlinear Programming (MINLP) problem, due to the use of index variables for channel assignment and logarithmic relationship between link capacity and signal to noise ratio (SNR). Such problems are NP-hard in general. We first propose a reformulation-linearization technique (RLT)-based centralized algorithm that computes near-optimal solutions in polynomial time [9]. We then develop a distributed greedy algorithm that uses only local information and computes near-optimal solutions. Through simulation studies, we find the distributed greedy algorithm outperforms both the RLT-based centralized algorithm and a heuristic channel assignment algorithm that exploits multiuser diversity with considerable gains.

The remainder of this paper is organized as follows. We describe the system model and preliminaries in Section 2. We present the problem formulation and develop the centralized and distributed algorithms in Section 3. Our simulation studies are shown in Section 4 and related work is discussed in Section 5. Section 6 concludes the paper. Throughout the paper, a symbol with a super-script "+" means a parameter related to the adjacent channel with a higher index; a symbol with a super-script "-" means a parameter related to the adjacent channel with a lower index.

\section{System model and preliminaries}

\subsection{Primary and $C R$ network model}

As shown in Fig. 1, we consider a primary network where a base station transmits data to primary users using $M$ licensed channels with non-overlapping spectrum. Without loss of generality, we assume the channels have identical bandwidth. We assume that each primary user is equipped with one transceiver and can communicate with the primary base station via one of the licensed channels. Let $\mathcal{P}_{m}$ be the subset of primary users that are tuned to channel $m$. All the $\mathcal{P}_{m}$ 's are generally assumed nonempty.

As in prior work $[2,10]$, we assume that the primary network uses a synchronous time slot structure. The occupancy of each channel can be modeled as a discrete-time Markov process. The status of channel $m$ in time slot $t$ is denoted by $S_{m}(t)$ : when the channel is idle, we have $S_{m}(t)=0$; when the channel is busy, we have $S_{m}(t)=1$. Let $P_{m}^{01}$ and $P_{m}^{10}$ be the transition probability from state 0 to 1 and that from state 1 to 0 for channel $m$, respectively. The utilization of channel $m$ with respect to primary user transmission, denoted by $\eta_{m}$, can be written as

$\eta_{m}=\operatorname{Pr}\left\{S_{m}(t)=1\right\}=\lim _{T \rightarrow \infty} \frac{1}{T} \sum_{t=1}^{T} S_{m}(t)=\frac{P_{m}^{01}}{P_{m}^{01}+P_{m}^{10}}$.

Within the coverage of the primary network, there are $K$ pairs of cognitive radio (CR) transmitters and receivers that explore the spectrum opportunities in the $M$ licensed channels for data communications. Each CR node is equipped with two transceivers: a control transceiver that operates on a dedicated control channel (which we assume is reliable as in prior work $[2,10])$, and a data transceiver incorporating a software defined radio (SDR) that is able to tune to any of the $M$ licensed channels.

$\mathrm{CR}$ nodes access the licensed channels following the same time slot structure as in the primary network. For CR nodes, each time slot consists of a sensing phase and a transmission phase. In the sensing phase, a CR node chooses one of the $M$ channels to sense using its data transceiver, and then exchanges the sensed channel information with other $C R$ nodes using its control transceiver over the control channel. During the transmission phase, the CR node tunes its data transceiver to one of the $M$ channels to transmit or receive data based on sensing results.

\subsection{Cooperative spectrum sensing}

We adopt a hypothesis test to detect the availability of each licensed channel. The null hypothesis and the alternative hypothesis are:

$$
\begin{cases}H_{0}^{m}: & \text { channel } m \text { isidle } \\ H_{1}^{m}: & \text { channel } m \text { isbusy. }\end{cases}
$$

During the sensing phase, the $C R$ nodes exchange their sensing results through the control channel. For the $n$th sensing result on channel $m$, we consider both types of sensing errors, i.e., false alarm with probability $\epsilon_{n}^{m}$ and miss detection with probability $\delta_{n}^{m}$, respectively. It has been shown that these sensing errors are inevitable and should be considered in CR networking protocol design [2,5]. We than have

$$
\left\{\begin{array}{l}
\epsilon_{n}^{m}=\operatorname{Pr}\left(\Theta_{n}^{m}=1 \mid H_{0}^{m}\right) \\
\delta_{n}^{m}=\operatorname{Pr}\left(\Theta_{n}^{m}=0 \mid H_{1}^{m}\right),
\end{array}\right.
$$

where $\Theta_{n}^{m}$ is the $n$th sensing result on channel $m$. Given $N$ sensing results on channel $m$, the conditional probability 


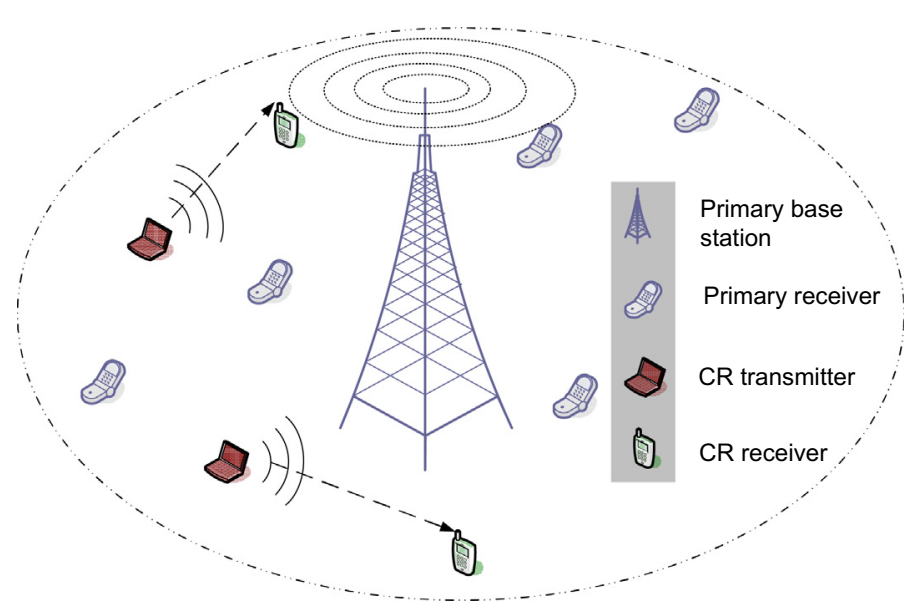

Fig. 1. The primary and $C R$ network model.

that channel $m$ is available, denoted by $P_{m}^{A}\left(\Theta_{1}^{m}, \cdots, \Theta_{N}^{m}\right)$, can be computed as [6]:

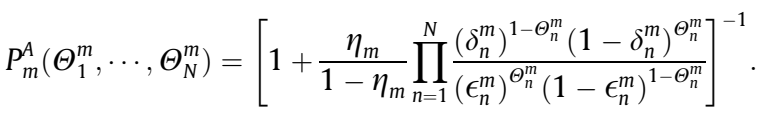

When one or more sensing results are received at a CR node, let the sensing result vector be $\vec{\Theta}_{n}^{m}=\left[\Theta_{1}^{m}, \cdots, \Theta_{n}^{m}\right]$ for the $n$ received sensing results on channel $m$. The conditional channel availability probability can be computed iteratively as follows.

$$
\begin{aligned}
& P_{m}^{A}\left(\Theta_{1}^{m}\right)=\left[1+\frac{\eta_{m}}{1-\eta_{m}} \times \frac{\left(\delta_{1}^{m}\right)^{1-\Theta_{1}^{m}}\left(1-\delta_{1}^{m}\right)^{\Theta_{1}^{m}}}{\left(\epsilon_{1}^{m}\right)^{\Theta_{1}^{m}}\left(1-\epsilon_{1}^{m}\right)^{1-\Theta_{1}^{m}}}\right]^{-1} \\
& P_{m}^{A}\left(\vec{\Theta}_{n}^{m}\right)=P_{m}^{A}\left(\Theta_{1}^{m}, \Theta_{2}^{m}, \cdots, \Theta_{n}^{m}\right) \\
& =\left\{1+\left[\frac{1}{P_{m}^{A}\left(\Theta_{1}^{m}, \Theta_{2}^{m}, \cdots, \Theta_{n-1}^{m}\right)}-1\right] \times \frac{\left(\delta_{n}^{m}\right)^{1-\Theta_{n}^{m}}\left(1-\delta_{n}^{m}\right)^{\Theta_{n}^{m}}}{\left(\epsilon_{n}^{m}\right)^{\Theta_{n}^{m}}\left(1-\epsilon_{n}^{m}\right)^{1-\Theta_{n}^{m}}}\right\}^{-1}, \\
& n=2, \cdots, N .
\end{aligned}
$$

\subsection{Opportunistic channel access}

Let $D_{m}(t)$ be a decision variable indicating whether channel $m$ will be accessed in time slot $t$. It is defined as

$D_{m}(t)= \begin{cases}0, & \text { if channel } m \text { is considered idle } \\ 1, & \text { otherwise }\end{cases}$

for $m=\{1,2, \cdots, M\}$.

Based on the spectrum sensing result $P_{m}^{A}\left(\vec{\Theta}_{N}^{m}\right)$, channel $m$ will be accessed (i.e., when $D_{m}(t)=0$ ) with probability $P_{m}^{D}\left(\vec{\Theta}_{N}^{m}\right)$, and it will not be accessed (i.e., when $D_{m}(t)=1$ ) with probability $1-P_{m}^{D}\left(\vec{\Theta}_{N}^{m}\right)$. We show how to compute $P_{m}^{D}\left(\vec{\Theta}_{N}^{m}\right)$ in the following.

For primary user protection, the probability that a $\mathrm{CR}$ transmission collides with primary user transmissions should be smaller with a threshold prescribed by the primary network, denoted by $\gamma_{m}$ for channel $m$. The primary user protection condition can be written as

$$
\left[1-P_{m}^{A}\left(\vec{\Theta}_{N}^{m}\right)\right] P_{m}^{D}\left(\vec{\Theta}_{N}^{m}\right) \leqslant \gamma_{m}
$$

To maximize the CR network throughput, $P_{m}^{D}\left(\vec{\Theta}_{N}^{m}\right)$ should be set to a probability as large as possible, as allowed by the maximum collision rate constraint. We have from (8)

$P_{m}^{D}\left(\vec{\Theta}_{N}^{m}\right)=\min \left\{\frac{\gamma_{m}}{1-P_{m}^{A}\left(\vec{\Theta}_{N}^{m}\right)}, 1\right\}$.

Let $\mathcal{A}(t):=\left\{m \mid D_{m}(t)=0\right\}$ be the subset of channels that are identified to be idle in time slot $t$. Then its complement set $\overline{\mathcal{A}}(t)$ is the subset of channels that are believed to be busy in time slot $t$ (i.e., being used by primary users). We next investigate how to effectively assign the channels in $\mathcal{A}(t)$ to the CR transmitters and how to choose transmit power for the CR transmitters, such that the CR network throughput is maximized under both interference and collision rate constraints.

\subsection{Channel interference model}

We consider Co-channel interference (CCI) among $\mathrm{CR}$ users sharing the same licensed channel and Adjacent channel interference (ACI) for both CR and primary users in this paper. The $\mathrm{CCI}$ and $\mathrm{ACl}$ models are presented in the following.

\subsubsection{Co-channel interference}

$\mathrm{CCI}$ is caused by the $\mathrm{CR}$ user transmissions sharing the same channel as the victim receiver. Before introducing the interference model, we define index variables $x_{k}^{m}$ indicating the channel assignment for the CR links as follows:

$x_{k}^{m}= \begin{cases}1, & \text { if channel } m \text { is used by CR link } k \\ 0, & \text { otherwise }\end{cases}$

$$
m=1, \cdots, M, k=1, \cdots, K .
$$

Let $\mathcal{T}_{k}$ and $\mathcal{R}_{k}$ be the transmitter and receiver of CR link $k$, respectively. The CCI at CR receiver $k$ on channel $m$, denoted by $C_{k}^{m}$, is 


$$
\begin{aligned}
C_{k}^{m} & =\sum_{i \in \Phi, i \neq k} G_{\mathcal{T}_{i}, \mathcal{R}_{k}}^{m} P_{i}^{m} x_{i}^{m} \\
& =\sum_{i \in \Phi} G_{\mathcal{T}_{i}, \mathcal{R}_{k}}^{m} P_{i}^{m} x_{i}^{m}-G_{\mathcal{T}_{k}, \mathcal{T}_{k}}^{m} P_{k}^{m} x_{k}^{m},
\end{aligned}
$$

where $G_{\mathcal{T}_{i}, \mathcal{R}_{k}}^{m}$ is the channel gain from CR transmitter $i$ to CR receiver $k$ on channel $m, P_{i}^{m}$ is the transmit power of CR transmitter $i$ on channel $m$, and $\Phi:=\{1,2, \cdots, K\}$ is the set of transmitter/receiver pairs in the CR network.

\subsubsection{Adjacent channel interference}

In addition to $\mathrm{CCI}$, a $\mathrm{CR}$ receiver may also be interfered by transmissions on an adjacent channel, when the channels are not strictly orthogonal. The interferer could be either a CR transmitter or a primary transmitter (e.g., the primary base station) on the adjacent channel. Such ACI is shown to be harmful with testbed experiments in a recent work [8].

Due to the imperfect design of band-pass filters, a portion of the power on the adjacent channel may leak to the channel being used by CR users. Such leakage is also considered as noise. For ease of explanation, we only consider the $\mathrm{ACI}$ from a direct neighboring channel in this paper. Let $\beta_{m}^{+}$be the ratio of leakage power from channel $(m+1)$ to $m$, and $\beta_{m}^{-}$the ratio of leakage power from channel $(m-1)$ to $m$. We term these leakage power ratios ACI factor, which depends on the spectral properties, such as inter-channel distance and channel width, and band-pass filter design.

For a channel $m$, if its adjacent channel $(m+1)$ is idle, then the $\mathrm{ACI}$ is due to the concurrent $\mathrm{CR}$ transmissions on channel $(m+1)$. We have

$$
\begin{aligned}
A C_{m, k}^{+} & =\left[1-D_{m+1}(t)\right] \beta_{m}^{+} \sum_{i \in \Phi, i \neq k} G_{\mathcal{T}_{i}, \mathcal{R}_{k}}^{m+1} P_{i}^{m+1} x_{i}^{m+1} \\
& =\left[1-D_{m+1}(t)\right] \beta_{m}^{+} C_{k}^{m+1} .
\end{aligned}
$$

Alternatively, if the adjacent channel $(m+1)$ is busy, then the $\mathrm{ACl}$ is caused by a primary transmission on channel $(m+1)$. We have

$A P_{m, k}^{+}=D_{m+1}(t) \beta_{m}^{+} G_{0, \mathcal{R}_{k}}^{m+1} Q_{m+1}$,

where $G_{0, \mathcal{R}_{k}}^{m+1}$ is the channel gain from the primary transmitter to $C R$ receiver $k$ on channel $(m+1)$, and $Q_{m+1}$ is the transmit power of the primary transmitter on channel $(m+1)$.

Similarly, ACI on channel $m$ may also come from the adjacent channel on the other side, i.e., channel $(m-1)$. We define $A C_{m, k}^{-}$and $A P_{m, k}^{-}$as the interference due to $C R$ transmission and primary transmission on channel $m-1$, respectively. These can be computed as

$A C_{m, k}^{-}=\left[1-D_{m-1}(t)\right] \beta_{m}^{-} C_{k}^{m-1}$

$A P_{m, k}^{-}=D_{m-1}(t) \beta_{m}^{-} G_{0, \mathcal{R}_{k}}^{m-1} Q_{m-1}$.

The total ACI on channel $m$ from its two adjacent channels can be written as

$A_{k}^{m}=A C_{m, k}^{+}+A P_{m, k}^{+}+A C_{m, k}^{-}+A P_{m, k}^{-}$.

Without loss of generality, we assume $A C_{1, k}^{-}, A P_{1, k}^{-}, A C_{M, k}^{+}$ and $A P_{M, k}^{+}$are all zero for channels 1 and $M$. This is because the adjacent channels 0 and $(M+1)$ are used by neither primary nor CR users.
On the other hand, primary users may also be interfered by $C R$ users transmitting on an adjacent channel. If channel $m$ is used by primary user $j$ and channel $(m+1)$ is available for CR user access, the ACI received by the primary user is

$B C_{m, j}^{+}=\left[1-D_{m+1}(t)\right] \beta_{m}^{+} \sum_{i \in \Phi} G_{\mathcal{T}_{i} j}^{m+1} P_{i}^{m+1} x_{i}^{m+1}$.

The ACI received by the primary user from CR transmissions on channel $(m-1)$ is

$B C_{m, j}^{-}=\left[1-D_{m-1}(t)\right] \beta_{m}^{-} \sum_{i \in \Phi} G_{\mathcal{T}_{i} . j}^{m-1} P_{i}^{m-1} x_{i}^{m-1}$.

Considering ACI from both sides of channel $m$, the total $\mathrm{ACI}$ at a primary receiver can be written as:

$B_{j}^{m}=B C_{m, j}^{+}+B C_{m, j}^{-}$.

Again, we assume $B C_{1, j}^{-}$and $B C_{M, j}^{+}$are zero for the two border channels 1 and $M$.

\section{Channel selection and power allocation}

\subsection{Problem statement}

At each cognitive radio $(\mathrm{CR})$ receiver, both types of interference from co-channel and adjacent channels are treated as noise. Let $v_{k}^{m}$ be the SNR at CR receiver $k$ on channel $m$. Then $v_{k}^{m}$ can be written as

$v_{k}^{m}=\frac{G_{\mathcal{T}_{k}, \mathcal{R}_{k}}^{m} P_{k}^{m} x_{k}^{m}}{N_{0}+C_{k}^{m}+A_{k}^{m}}$,

where $N_{0}$ is the channel noise power. The objective is to maximize the capacity of the CR network as approximated by Shannon capacity. Without loss of generality, we assume that each channel has unit bandwidth. The objective function becomes

$\max _{P_{k}^{m}, x_{k}^{m}} \sum_{k \in \Phi} \sum_{m \in \mathcal{A}(t)} \log _{2}\left(1+v_{k}^{m}\right)$.

Since each CR user is able to access one channel in each time slot, we have the following channel access constraint.

$\sum_{m \in \mathcal{A}(t)} x_{k}^{m} \leqslant 1, \quad$ for all $k \in \Phi$.

Furthermore, each CR transmitter is limited by a peak power constraint. That is

$\sum_{m \in \mathcal{A}(t)} P_{k}^{m} x_{k}^{m} \leqslant \Gamma, \quad$ for all $k \in \Phi$.

As discussed, the interference from $\mathrm{CR}$ transmissions to primary users should be bounded. Recall that $\overline{\mathcal{A}}(t)$ is the set of busy channels and $\mathcal{P}_{m}$ is the set of primary users using channel $m$. Letting the ACI bound be $\Omega$, we have

$B_{j}^{m} \leqslant \Omega, \quad$ for all $m \in \overline{\mathcal{A}}(t), j \in \mathcal{P}_{m}$.

Problem (21) with constraints (22)-(24) maximizes the CR network capacity while bounding the total interference (i.e., both common channel interference (CCI) and adjacent channel interference $(\mathrm{ACI})$ ) to primary users. Note that the maximum collision rate constraint caused by CR transmissions (8) is satisfied by choosing the channel $m$ access 
probability $P_{m}^{D}\left(\vec{\Theta}_{N}^{m}\right)$ as in (9). Based on spectrum sensing results, we need to determine channel access (as given by the $x_{k}^{m}$ 's) as well transmit powers (as given by the $P_{k}^{m}$ 's) for CR users. This is an MINLP problem, which is NP-hard in general and cannot be solved exactly in polynomial time. For this problem, we first describe below how to derive upper and lower bounds with a centralized algorithm, and then present a distributed algorithm that decomposes Problem (21) into a channel assignment subproblem and a power allocations subproblem in the next section.

\subsection{Centralized Algorithm and Performance Bounds}

In this section, we first obtain an upper bound by relaxing the problem with RLT [9]. The lower bound is then computed with a sequential fixing (SF) algorithm [11,12]. To obtain a linear relaxation of the MINLP problem, we first allow the binary variables $x_{k}^{m}$ to take real values in $[0,1]$. Second, the product term $P_{k}^{m} x_{k}^{m}$ is replaced by a substitution variable $\phi_{k}^{m}=P_{k}^{m} x_{k}^{m}$. Since $0 \leqslant P_{k}^{m} \leqslant \Gamma$ and $0 \leqslant x_{k}^{m} \leqslant 1$, we derive the following RLT bound-factor product constraints.

$\left\{\begin{array}{l}\left(P_{k}^{m}-0\right)\left(x_{k}^{m}-0\right) \geqslant 0 \\ \left(P_{k}^{m}-0\right)\left(1-x_{k}^{m}\right) \geqslant 0 \\ \left(\Gamma-P_{k}^{m}\right)\left(x_{k}^{m}-0\right) \geqslant 0 \\ \left(\Gamma-P_{k}^{m}\right)\left(1-x_{k}^{m}\right) \geqslant 0\end{array}\right.$

Rearranging the terms, we have

$$
\left\{\begin{array}{l}
\phi_{k}^{m} \geqslant 0 \\
P_{k}^{m}-\phi_{k}^{m} \geqslant 0 \\
\Gamma x_{k}^{m}-\phi_{k}^{m} \geqslant 0 \\
P_{k}^{m}+\Gamma x_{k}^{m}-\phi_{k}^{m} \leqslant \Gamma .
\end{array}\right.
$$

Finally, the logarithm term $\log _{2}\left(1+v_{k}^{m}\right)$ in the objective function can be decomposed into the difference between two logarithm terms, denoted by $y_{k}^{m}$ and $z_{k}^{m}$, respectively, as follows.

$$
\begin{aligned}
\log _{2}\left(1+v_{k}^{m}\right)= & \log _{2}\left(N_{0}+C_{k}^{m}+A_{k}^{m}+G_{\mathcal{T}_{k}, \mathcal{R}_{k}}^{m} P_{k}^{m} x_{k}^{m}\right) \\
& -\log _{2}\left(N_{0}+C_{k}^{m}+A_{k}^{m}\right) \\
= & y_{k}^{m}-z_{k}^{m}
\end{aligned}
$$

where $\quad y_{k}^{m}:=\log _{2}\left(N_{0}+C_{k}^{m}+A_{k}^{m}+G_{\mathcal{T}_{k}, \mathcal{R}_{k}}^{m} P_{k}^{m} x_{k}^{m}\right) \quad$ and $z_{k}^{m}:=\log _{2}\left(N_{0}+C_{k}^{m}+A_{k}^{m}\right)$. For a general logarithm term $\log _{2}(x)$, we can linearize it over some tightly bounded regions with a polyhedral outer approximation. For example, if $x$ is bounded by $x_{0} \leqslant x \leqslant x_{L}$, we can determine $L$ evenly spaced points as

$\left.x_{l}=x_{0}+\frac{l}{L}\left(x_{L}-x_{0}\right),\right\}$ for $\mathrm{l}=0,1, \mathrm{~L}$.

Then the logarithmic function $y=\log _{2}(x)$ can be substituted with the following linear constraints.

$$
\left\{\begin{array}{l}
y \geqslant \frac{\log _{2}\left(x_{L}\right)-\log _{2}\left(x_{0}\right)}{x_{L}-x_{0}}\left(x-x_{0}\right)+\log _{2}\left(x_{0}\right) \\
y \leqslant \frac{1}{\ln (2) x_{l}}\left(x-x_{l}\right)+\log _{2}\left(x_{l}\right), \text { for } l=0, \cdots, L .
\end{array}\right.
$$

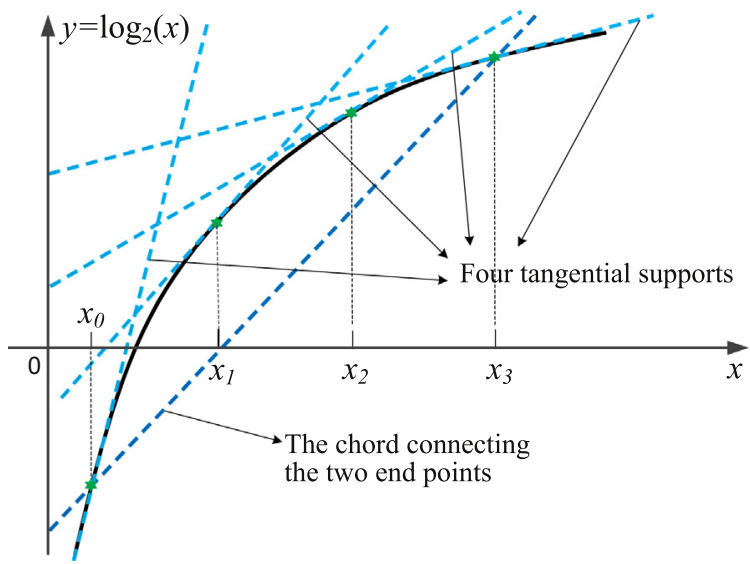

Fig. 2. The polyhedral outer approximation of a logarithm function $y=\log _{2}(x)$ in $x_{0} \leqslant x \leqslant x_{3}$.

In this paper, we use a four-point (i.e., $L=3$ ) tangential approximation. ${ }^{1}$ The upper and lower bound of $y_{k}^{m}$ and $z_{k}^{m}$ can be obtained by letting $\phi_{k}^{m}$ be 0 and $\Gamma$, respectively. The five linear constraints given in (29) that form the polyhedral outer approximation are plotted in Fig. 2.

With the above three-step relaxations, we thus obtain a linear programming (LP) relaxation for Problem (21). Solving the LP relaxation with an LP solver, we can obtain a possibly infeasible solution due to the relaxations, which can serve as an upper bound for the original problem.

We next present a sequential fixing (SF) Algorithm in Algorithm 1 for deriving a feasible near-optimal solution. In Steps 3-9, the variable $x_{k^{\prime}}^{m^{\prime}}$ that is closest to 0 or 1 is chosen and rounded to the nearest binary integer. Once $x_{k^{\prime}}^{m^{\prime}}$ is fixed to 1 , all the other variables $x_{k^{\prime}}^{m}$ with the same subscript $k^{\prime}$ are fixed to 0 , due to constraint (22). Then the problem can be reformulated with a reduced size, and solved again iteratively, until all the binary variables $x_{k}^{m}$ 's are fixed. In Step 16 , the transmit powers $P_{k}^{m}$ 's are derived when the channel assignment is determined. Note that here we still need to formulate and solve an $\mathrm{LP}$ relaxation with respect to the logarithmic terms, since even when the binary variables are fixed, the problem is still non-convex. Finally, in Step 17, we substitute the near-optimal feasible solution into the original objective function (21) to obtain a lower bound for the global optimum.

\footnotetext{
1 It is easy to see that the three-point tangential approximation is the minimum requirement (i.e., $L \geqslant 2$ ) to bound a logarithm curve with the resulting polygon. Usually, a larger $L$ provides a tighter polygon and thus achieves a smaller gap between the upper and lower bounds. However, a larger $L$ will introduce more linear constraints (see Eq. (29)) for each logarithm term and increases the computational complexity. So there is actually a tradeoff here. In our prior work [9], we show that this approach can be incorporated into the branch-and-bound algorithm to iteratively tighten the approximation, but at a higher computational complexity. In our numerical studies, and also according to our previous experience with this technique, $L=3$ is sufficient for good results.
} 


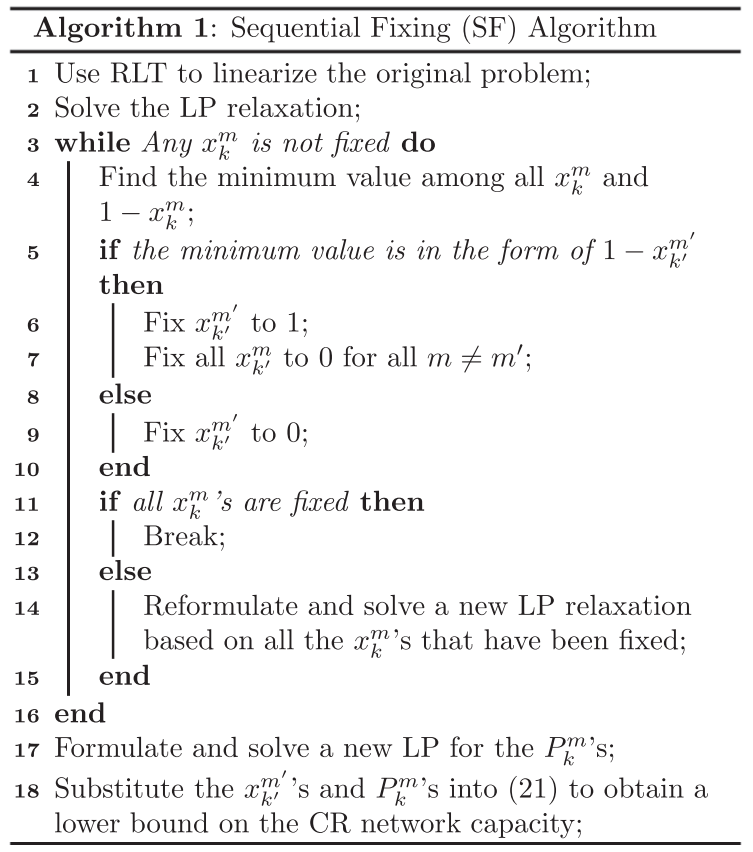

Generally, solving such an LP relaxation may produce an infeasible solution to the original problem due to the relaxations; a local search algorithm is then needed to find a feasible solution in the neighborhood. However, local search is not necessary in our problem. First, the SF algorithm determines binary values for all the $x_{k}^{m}$ 's, such that constraint (10) is satisfied. Second, from the RLT boundfactor product constraints (26), we have that $\phi_{k}^{m}=P_{k}^{m}$ when $x_{k}^{m}=1$; and $\phi_{k}^{m}=0$ when $x_{k}^{m}=0$. Once all the $x_{k}^{m}$ 's are set to binary values, by replacing $\phi_{k}^{m}$ with $P_{k}^{m}$ or 0 , the linear inequality constraints now only contain the $P_{k}^{m}$ 's. Finally, although we use the polyhedral outer approximation for the logarithmic terms, these terms are all in the objective function (21). It is easy to show that the feasible solutions of the relaxed LP problem is a subset of the feasible solution of the original problem, since the constraints of the original problem is a subset of those of the relaxed LP problem. Therefore, a feasible problem to the LP relaxation is also feasible to the original problem. We only need to substitute the feasible solution into the original objective function (21) to obtain the corresponding objective value, which is a lower bound for the original problem.

In our simulations, we find the upper bound quite loose, but the lower bound is reasonably tight. The average-case time complexity of the simplex method, a popular LP solving algorithm, is $O(n \log n)$ for a problem with size $n$ [13]. Thus the computational complexity of one iteration in SF is $O(M K \log (M K))$. Since the number of iterations in SF is $M K$ in the worst case, the overall average-case computational complexity of SF is $O\left(M^{2} K^{2} \log (M K)\right)$.

It is also worth noting that the centralized scheme is mainly used as a benchmark for comparison purpose. In Section 4, we compare the centralized scheme with the proposed distributed algorithm (see Section 3.3) to validate the performance of the latter. We assume a dedicated control channel and all the channel information are cor- rectly delivered when executing the centralized algorithm. In a real deployment, the control channel may introduce errors and the performance of the centralized algorithm may be worse.

\subsection{Distributed algorithm}

\subsubsection{Distributed algorithm}

Although the SF algorithm in Algorithm 1 can compute a near-optimal solution in polynomial time, it is a centralized algorithm that needs to know all the channel gains. In this section, we present a distributed greedy algorithm for solving Problem (21). With this algorithm, each CR transmitter estimates channel gains from itself to primary users and all other $C R$ receivers, and each $C R$ receiver estimates channel gains from the primary base station and all other CR transmitters.

The distributed algorithm consists of two tiers: (i) the upper tier is a channel assignment algorithm, which decides which channel to access for a CR transmitter, and (ii) the low tier is a power allocation algorithm, which decides how much power can be allocated to transmit on each available channel. In the channel assignment algorithm, we assume that the transmit powers have already been allocated to each available channel; the power allocation, denoted by an $M \times K$ vector $\vec{P}$, can be obtained from the power allocation algorithm. Define the capacity of CR link $k$ if it uses channel $m$ as

$U_{k}^{m}=\log _{2}\left(1+v_{k}^{m}\right)$.

Then in each loop, the channel with the lowest $U_{k}^{m}(\vec{P})$ is removed from the available channel set $\mathcal{A}_{k}(t)$ and the corresponding $x_{k}^{m}$ is set to 0 , until only one available channel is left.

The complete channel assignment algorithm is presented in Algorithm 2. With the algorithm, initially we assume CR transmitter $k$ uses all the channels in $\mathcal{A}(t)$ in Step 1. Then in Steps 2-6, we iteratively remove the channels with the minimum capacity gain, until only one channel is left. Finally, the transmit power is determined for the chosen channel in Step 7. The loop (i.e., Steps 2-6) in the channel allocation algorithm is executed at most $M$ times in the worst case, where $M$ is the number of channels.

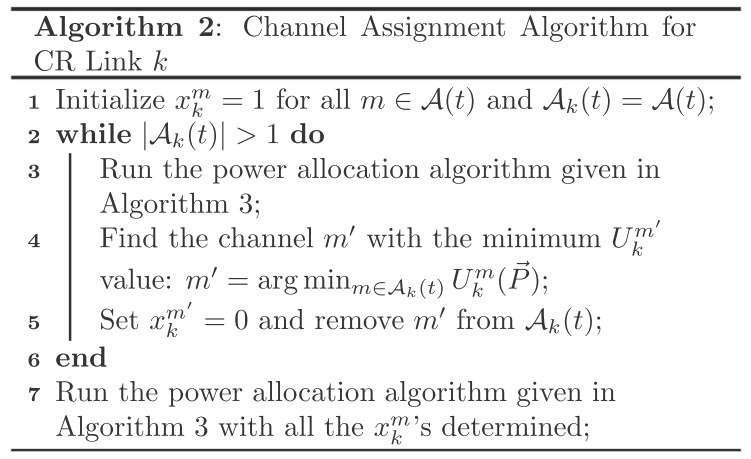

In the power allocation algorithm, the main idea is to iteratively allocate a small amount of power $\Delta$ to the CR link that can achieve the largest increase in (21). The algorithm is presented in Algorithm 3. Let $\vec{\Delta}_{k}^{m}$ be a vector whose $[(k-1) \times M+m]$ th element is $\Delta$ and all other elements are 0 , indicating that power $\Delta$ is allocated to CR link $k$ on 
channel $m$. Obviously, if CR link $k$ is allocated with the additional power $\Delta$, the throughput of this CR link will increase; if another CR link $k^{\prime} \neq k$ is allocated with the additional power $\Delta$, the throughput of CR link $k$ will decrease. The increase and decrease of the throughput of the CR link are defined as:

- $\mathcal{E}_{k}^{m}$ : the throughput increase for CR link $k$ on channel $m$ if it gets the additional power $\Delta$

- $\mathcal{C}_{k^{\prime}, k}^{m}$ : the throughput decrease for CR link $k$ if another CR link $k^{\prime} \neq k$ on channel $m$ wins the additional power allocation $\Delta$.

In Steps 3-8, we calculate $\mathcal{E}_{k}^{m}$, but set it to 0 if either (23) or(24) is not satisfied. In Steps 11-12, the net throughput gains of all the possible power allocations are evaluated and the combination with the largest profit is selected. We assume the control information (i.e., $\mathcal{E}_{k}^{m}$ and $\mathcal{C}_{k^{\prime}, k}^{m}$ ) are broadcast over orthogonal sub-channels, which means all CR users are able to broadcast the information simultaneously. In Steps 13-15, the CR link with the largest positive profit wins the additional power allocation $\Delta$, if its profit is positive. Otherwise, the power allocation algorithm is terminated with solution $\vec{P}$, because no further power allocation can improve the total throughput. The loop (i.e., Steps 3-16) in the power allocation algorithm is executed at most $\Gamma / \Delta$ times in the worst case (recall that $\Gamma$ is the peak power constraint). Since the overall distributed algorithm is a greedy algorithm, it will stop within a finite number of steps.

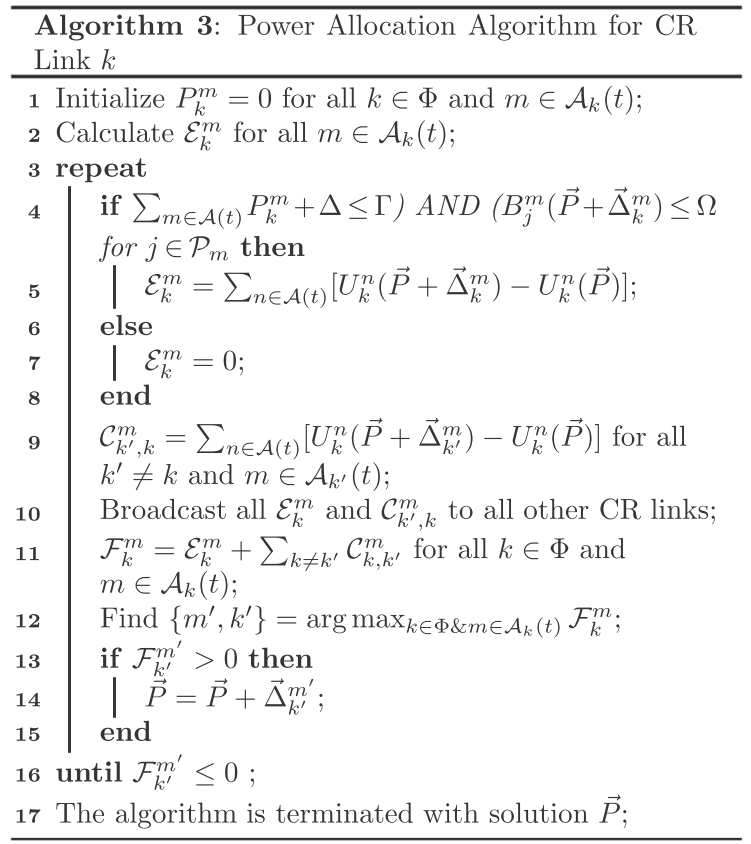

\subsubsection{A simple example}

To better explain the distributed algorithm, consider a simple example with two CR users 1 and 2. As shown in Fig. 3, for each channel $m$, CR transmitter 1 calculates $\mathcal{E}_{1}^{m}$ and $\mathcal{C}_{2,1}^{m}$ and $\mathrm{CR}$ transmitter 2 calculates $\mathcal{E}_{2}^{m}$ and $\mathcal{C}_{1,2}^{m}$. The

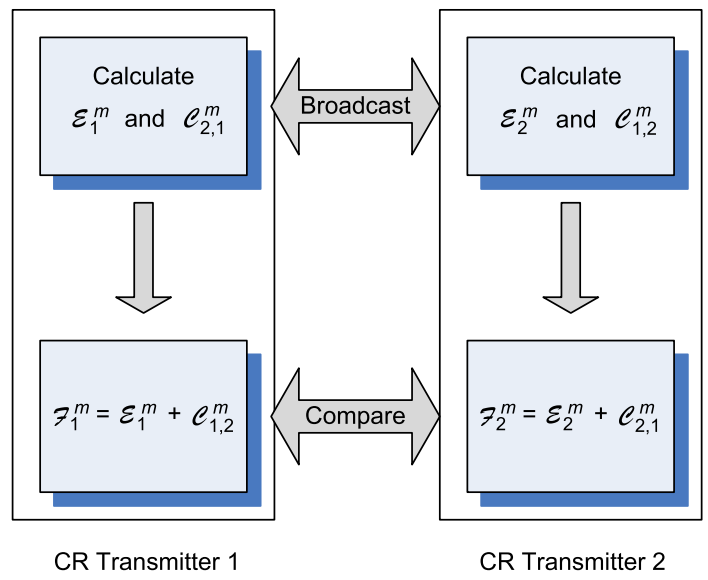

Fig. 3. An example of the distributed algorithm operation with two $C R$ users.

two nodes then broadcast the values to each other. Once a node receives the throughput decrease (i.e., $\mathcal{C}$ ) from the other node, it calculates its gain $\mathcal{F}$ as shown in the figure. The power unit $\Delta$ is allocated to the node with the larger $\mathcal{F}_{k}^{m}$ in this iteration. This power allocation algorithm is terminated if both $\mathcal{F}_{k}^{m}$ from the two nodes are non-positive. Once the power allocation algorithm is terminated, the channel with the lowest $U_{k}^{m}(\vec{P})$ is removed from the available channel set $\mathcal{A}_{k}(t)$, until only one available channel is left, as given in Algorithm 2.

With the distributed algorithm, the computational complexity is quite low since each node just makes some simple update of the three variables in each iteration, as shown in Algorithm 3.

\section{Performance evaluation}

\subsection{Simulation methodology}

As can be seen in the problem statement, the problem addressed in this paper is a Mixed Integer Nonlinear Programming (MINLP) problem, which is NP-hard. For such a complex problem, the main contribution of this paper is to develop algorithms that can provide highly competitive solutions. Since generally an efficient analysis is not feasible for this problem, we validate the performance of the proposed algorithms by comparisons with upper and lower bounds and alternative approaches with simulations.

For the results reported in this section, there are $M=6$ licensed channels (unless otherwise specified) with identical transition probabilities $P_{m}^{01}=0.4$ and $P_{m}^{10}=0.3$ for all $m$. The maximum collision probability with primary users is $\gamma_{m}=0.2$ for all $m$. The transmit power of primary base station is $30 \mathrm{dBm}$ and the maximum acceptable interference for the primary users is $\Omega=10 \mathrm{dBm}$. There are $K=6$ transmitter and receiver pairs in the $\mathrm{CR}$ network. The power of CR transmitter is limited to $\Gamma_{m}=27 \mathrm{dBm}$ for all $m$. The false alarm probability is $\epsilon_{n}^{m}=0.3$ and the miss detection probability is $\delta_{n}^{m}=0.3$ for all $m$ and $n$, unless otherwise 
specified. Rayleigh block fading channels are used in the simulations.

We consider four types of results:

- The upper bound obtained by solving the RLT relaxation as presented in Section 3.2.

- The centralized sequential fixing (SF) algorithm solution given in Algorithm 1 (a lower bound).

- The distributed greedy algorithm given in Algorithms 2 and 3.

- A simple centralized heuristic algorithm, where each CR transmitter chooses the best available channel to access to exploit diversity gain. When the channels are assigned and all the $x_{k}^{m}$ 's are fixed, it then solves the simplified problem (21) with MATLAB Optimization Toolbox to find a near-optimal power allocation.

We run each simulation for 10 times, each with a different random seed and each runs for a sufficiently long period of time until stable results are obtained. That is, each point in the curves is the average of 10 simulations. This approach allows us to compute the confidence intervals for the simulation results. The computed 95\% confidence intervals are plotted as error bars in the figures, which are all quite small in all the figures. Such small confidence intervals make the simulation results credible.

\subsection{Simulation results and discussions}

We first examine the impact of the number of channels $M$ on the overall throughput of the CR network. In Fig. 4, we increase $M$ from 4 to 8 , and plot the total throughput of the CR network. As expected, the more licensed channels, the more spectrum opportunities are available for the $\mathrm{CR}$ users. Thus the $\mathrm{CR}$ network throughput increases with $M$. The curves of both SF and the heuristic algorithm have lower slop than that of the distributed greedy algorithm. It implies that the greedy algorithm is more efficient in exploiting the addition spectrum opportunities for CR transmissions, since it achieves large throughput gain for an additional licensed channel. We find the upper bound quite loose, while the lower bound is reasonably tight.

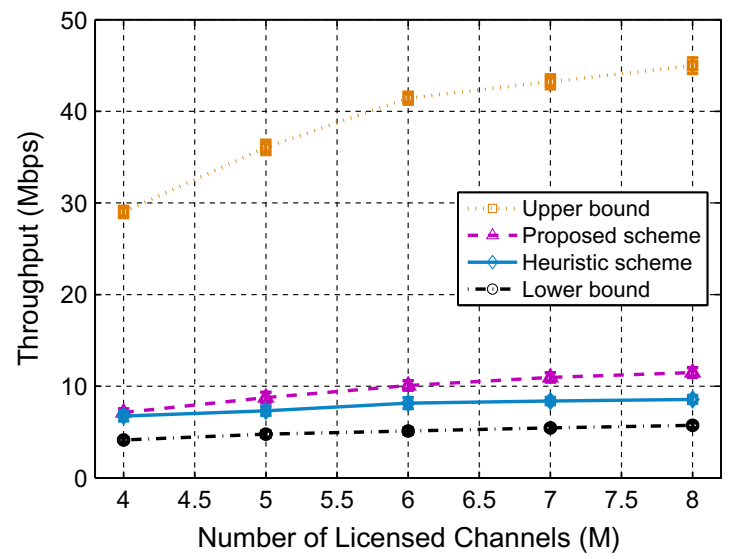

Fig. 4. CR network throughput versus the number of licensed channels.
Therefore we omit the upper bound in the following figures.

In Fig. 5, we investigate the impact of primary user channel utilization $\eta$ on the CR network throughput. The throughput curves achieved by the algorithms are plotted when $\eta$ is increased from 0.3 to 0.7 . Clearly, a smaller $\eta$ allows more spectrum opportunities for CR transmissions. When the primary users get more busy, the spectrum opportunities for CR users decreases and the throughput of all the three algorithms decreases. It can be seen from the figure that all the three curves decrease as $\eta$ gets larger. The CR network throughput of the distributed greedy algorithm is better than that of the simple heuristic algorithm and that of the centralized SF algorithm. In particular, when $\eta=0.3$, the distributed greedy algorithm achieves a normalized throughput gain of $27.84 \%$ over the simple heuristic algorithm, and a normalized throughput gain of $117.62 \%$ over SF. When $\eta=0.7$, the distributed algroithm achieves normalized throughput gains of $19.98 \%$ and $73.67 \%$ over the simple heuristic and SF, respectively.

Next we examine the impact of spectrum sensing errors on the CR network throughput. Since such sensing errors are generally unavoidable [2], it would be desirable for the $\mathrm{CR}$ network protocols to be robust to such errors. In Fig. 6, we test five pairs of $\{\epsilon, \delta\}$ values as follows: $\{0.2,0.48\}, \quad\{0.24,0.38\}, \quad\{0.3,0.3\}, \quad\{0.38,0.24\}$, and $\{0.48,0.2\}$. The CR network throughputs achieved by the algorithms are plotted in the figure. It is interesting to see that the throughput performance gets worse when the probability of one of the two sensing errors gets large. We can trade-off between false alarm and miss detection probabilities to find the optimal operating point for spectrum sensing. Again, the throughput performance of the greedy algorithm is superior to that of the heuristic algorithm and doubles that of the SF algorithm.

We then investigate the impact of the ACI factor $\beta$ on the $C R$ network throughput. The simulation results are presented in Fig. 7, where $\beta$ is increased from 0 to 0.5 . As expected, the CR network throughput is degraded by the presence of $\mathrm{ACI}$. The severer the $\mathrm{ACI}$, the lower the CR network throughput. When $\beta$ is increased from 0 to 0.5 , the throughput degradations are 4.0647 Mbps, 3.8068 Mbps,

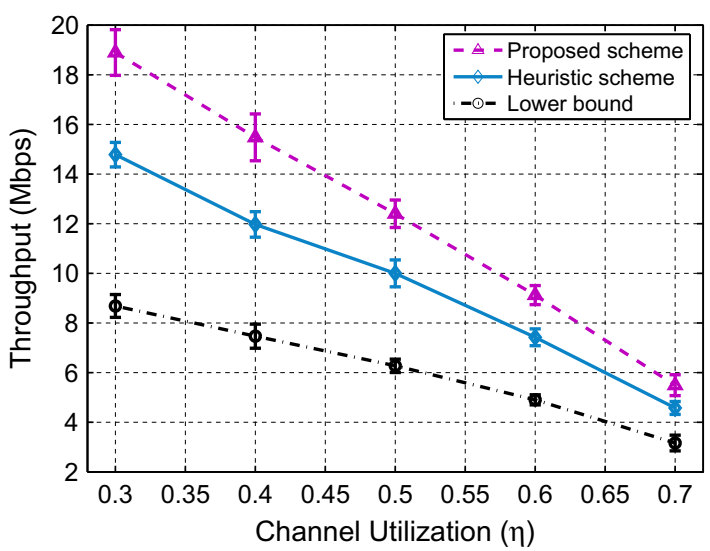

Fig. 5. CR network throughput versus primary user channel utilization. 


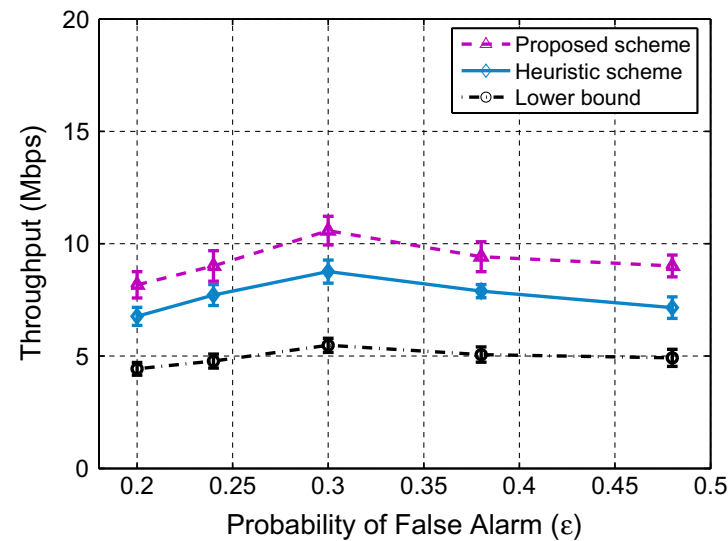

Fig. 6. $C R$ network throughput versus spectrum sensing error probabilities.

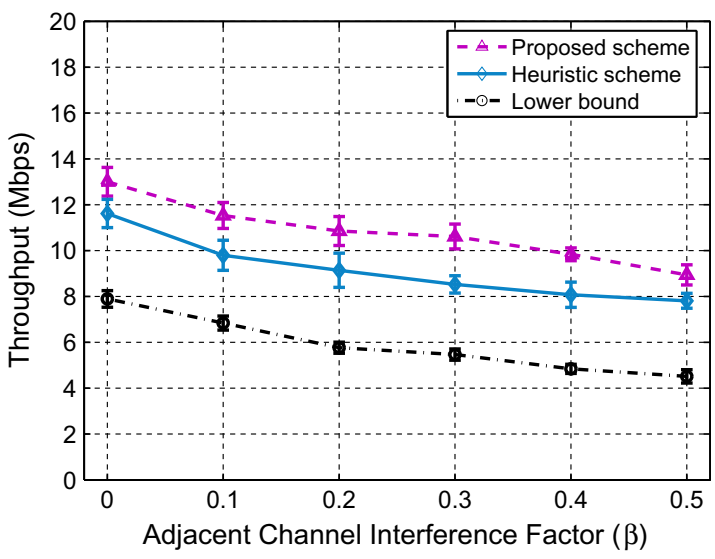

Fig. 7. CR network throughput versus the ACI factor.

and $3.3793 \mathrm{Mbps}$ for the distributed algorithm, the simple heuristic, and SF, respectively. The distributed greedy algorithm outperforms both the simple heuristic algorithm and SF with considerable gaps for the entire range of $\beta$ considered.

We also measure both types of interference in the simulations and exam the impact of the $\mathrm{ACI}$ factor $\beta$ on channel interference. In Fig. 8, we increase $\beta$ from 0 to 0.5 with step 0.1 and plot the measured average interference in the plots. The total average interference for each licensed channel is shown in Fig. 8a, which consists of both ACI and CCI. It can be seen that the total interference increases as $\beta$ gets larger, since there is more power leakage from adjacent channels. The ACI and CCI components are plotted in Fig. 8b and c, respectively. It can be seen that $\mathrm{ACI}$ almost linearly increases with $\beta$. When $\beta=0, \mathrm{ACI}$ is zero for all the three schemes since there is no power leakage from neighboring channels. When $\beta=0.5$, the $\mathrm{ACI}$ of the proposed distributed scheme is about $92.32 \%$ of that of the simple heuristic and $58.93 \%$ of that of SF. The proposed distributed algorithm curve has the lowest slop among the three schemes, indicating more effective control of ACI as $\beta$ increases. The fractions of $\mathrm{ACI}$ in the total average interference are plotted in Fig. $8 \mathrm{~d}$ for the three schemes. The fraction increases as $\beta$ gets larger, from $0 \%$ to about $22 \%$. Clearly ACI should be considered in the resource allocation and protocol design of CR networks; the proposed approach of jointly considering $\mathrm{CCI}$ and $\mathrm{ACI}$ is well justified.

Finally, we validate our proposed spectrum sensing and access scheme. We set the maximum allowable collision probability $\gamma$ to be 0.2 and increase channel utilization $\eta$ from 0.3 to 0.7 in steps of 0.1 . In Fig. 9, the measured collision rates with primary uses are plotted, along with the $\gamma=0.2$ curve. It can be seen that the measured collision rate is always kept below $\gamma$, showing that the proposed spectrum sensing and access scheme is quite effective with regard to primary user protection.

The trace of maximum profit, i.e., $\mathcal{F}$, achieved by the distributed algorithm from one of the simulations is plotted in Fig. 10. It can be seen that the distributed power allocation algorithm converges within about 167 steps, while in each step each node only updates and broadcasts several local variables (i.e., $\mathcal{E}, \mathcal{C}$, and $\mathcal{F}$ ). The computational complexity and the total amount of control data exchanged over the control channel are both quite low.

\section{Related work}

Cognitive radio (CR) has been recognized as a promising technology for efficient spectrum sharing [2,3]. There is considerable $\mathrm{CR}$ research on spectrum sensing and dynamic spectrum access $[5,10,14,15]$. The approach of periodically sensing a selected subset of channels has been adopted in the design of CR MAC protocols [5,10,15]. Several papers consider sensing errors in the design of spectrum access schemes [5,16,17]. The design of opportunistic channel access scheme was considered in $[15,16]$. Power allocation for CR users was one of the active research topics in CR networking. In a recent work [18], Zhao and Kwak investigated the power allocation problem for a single secondary user, while considering the mutual interference between primary users and secondary users.

Co-channel and adjacent channel interference (CCI and $\mathrm{ACI}$ ) are the two major factors limiting wireless network capacity. The impact of CCI on network performance is well-known and comprehensively investigated in [19]. Recently, the impact of ACI has attracted considerable interest in the wireless community. In $[20,8]$, the need was demonstrated for careful channel selection to mitigate ACI in IEEE 802.11 based systems. The impact of both CCI and $\mathrm{ACI}$ on network throughput and performance was evaluated in [21-23]. The interference models have been developed to analyze the channel interference in a few papers. In [24], the problem of statistical-physical modeling of CCI was investigated to analyze the outage probabilities in wireless networks and to design interference-aware transceivers. ACI was described by a simple quantification model that was verified by testbed experiments in [8]. In [25], a model for the aggregate ACI in TV white space was developed to demonstrate that the weighted sum of the total ACI power should be kept below certain threshold as well as ACI in each adjacent channel.

A commonly used approach to reduce $\mathrm{CCI}$ is to assign different channels to neighboring transmitters [6,7]. In 


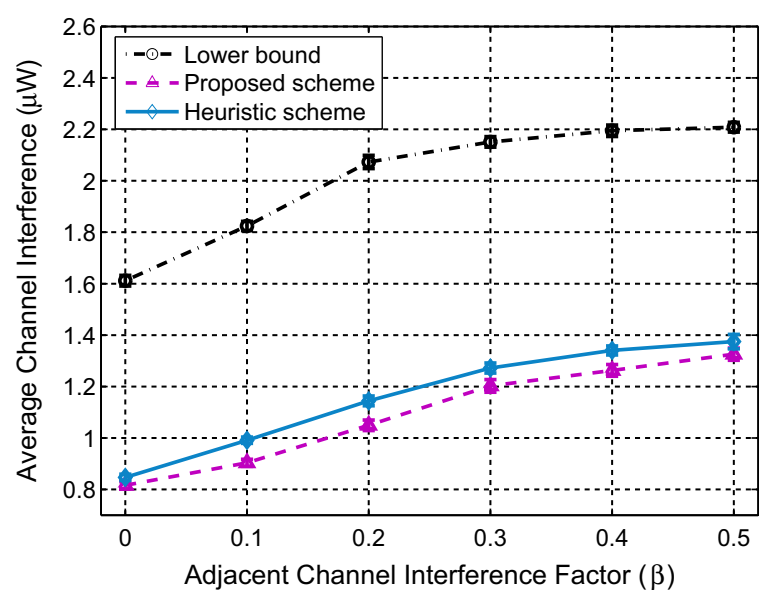

(a) Average total interference on a channel

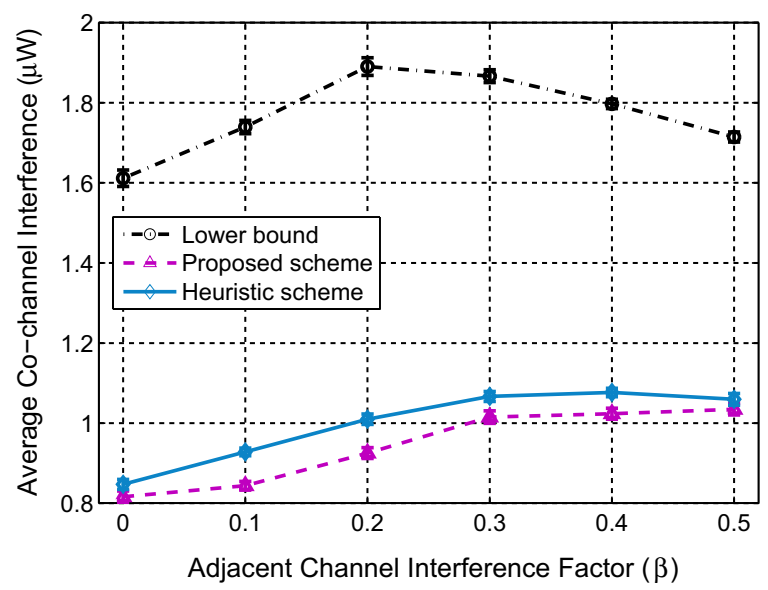

(c) Average CCI on a channel

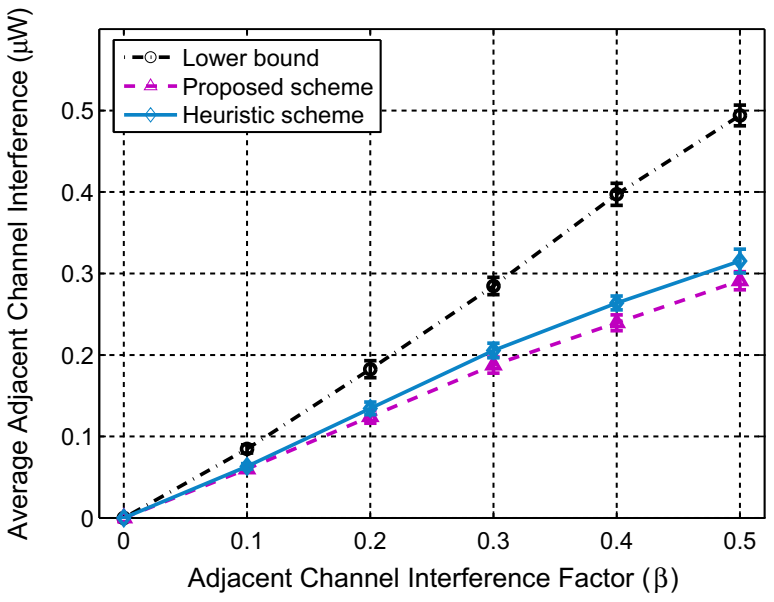

(b) Average ACI on a channel

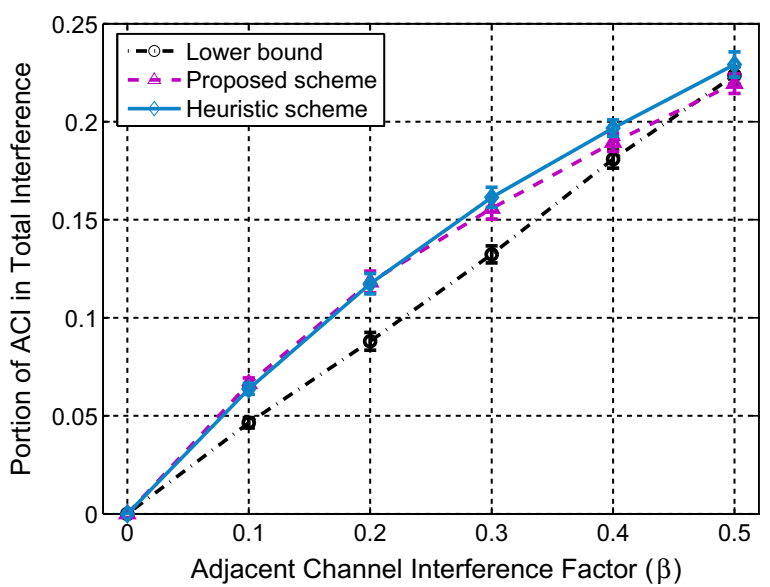

(d) Portion of ACI in the total interference

Fig. 8. Composition of the total interference measured in the simulations as a function of $\mathrm{ACI}$ factor $\beta$.

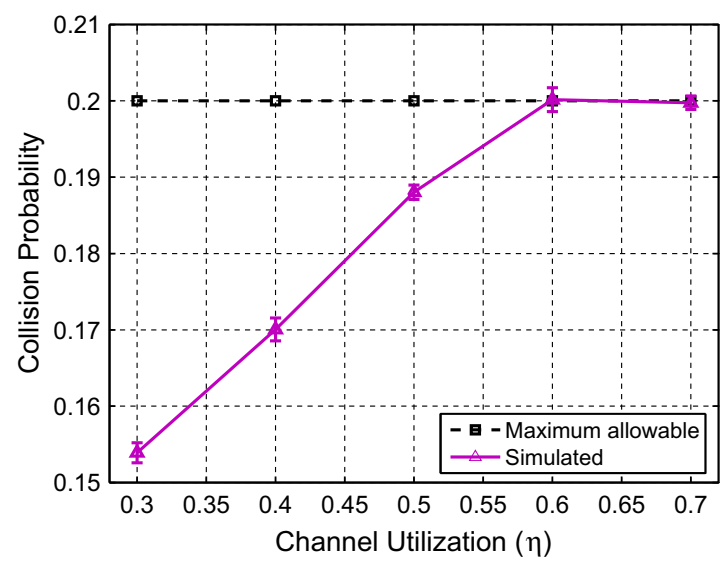

Fig. 9. Collision probability to primary users measured in the simulation.

[26,27], frequency domain iterative multi-user detectors were adopted for $\mathrm{CCI}$ cancelation. A low-cost CCI avoidance MAC scheme was presented in [28]. In [29], ACI was min-

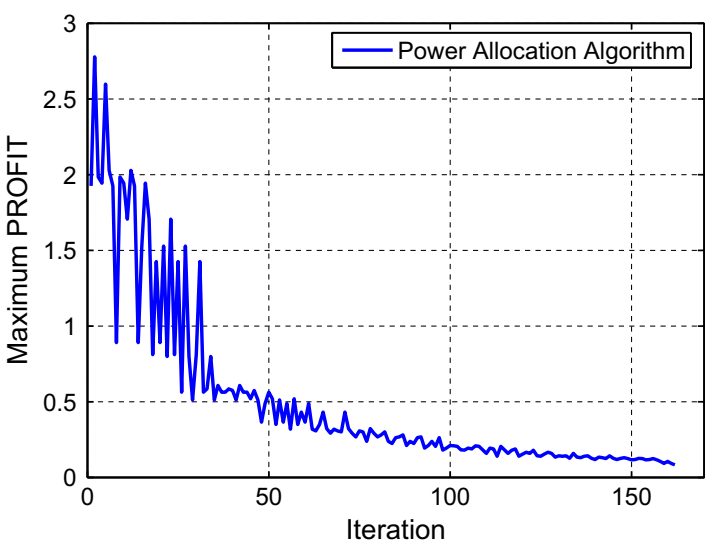

Fig. 10. Convergence of the maximum profit achieved by the distributed algorithm.

imized by optimizing reception of television receivers. In [30], Gidony and Kalet addressed the ACI mitigation problem by exploiting antenna diversity. 
In this paper, we consider the challenging problem of channel assignment and power allocation in $\mathrm{CR}$ networks under the presence of both $\mathrm{CCI}$ and $\mathrm{ACI}$, aiming to maximize the overall CR network capacity without imposing severely harmful impact on the primary users. We propose an RLT-based centralized SF algorithm and a distributed greedy algorithm for near-optimal channel assignment and power allocations. The proposed algorithms are shown to perform well in achieving the design goals.

\section{Conclusion}

In this paper, we investigated the problem of co-channel interference $(\mathrm{CCI})$ and adjacent channel interference (ACI) mitigation via channel assignment and power allocation in cognitive radio $(\mathrm{CR})$ networks. The objective was to maximize the total CR network throughput while keeping both collision rate and interference with primary users below tolerance thresholds. We proposed a reformulationlinearization technique (RLT)-based centralized sequential fixing (SF) algorithm that computes near-optimal solutions, and a distributed greedy algorithm that only uses local channel gain information. The proposed algorithms are evaluated with simulations. The distributed greedy algorithm is shown to outperform both the centralized SF algorithm and a centralized heuristic algorithm with considerable gains. In this paper we assumed reliable control channels for the CR users to exchange control information. The design of such a control channel and its impact on the $\mathrm{CR}$ network capacity are interesting problems to be investigated in the future work.

\section{Acknowledgments}

This work is supported in part by the US National Science Foundation (NSF) under Grants CNS-0953513 and CNS-1247955, and through the NSF I/UCRC Broadband Wireless Access and Application Center (BWAC) Site at Auburn University.

\section{References}

[1] D. Hu, S. Mao, Co-channel and adjacent channel interference mitigation in cognitive radio networks, in: Proc. IEEE MILCOM'11, Baltimore, MD, November 2011, pp. 1-6.

[2] Q. Zhao, B.M. Sadler, A survey of dynamic spectrum access, IEEE Signal Process. Mag. 24 (3) (2007) 79-89. May.

[3] Y. Zhao, S. Mao, J.O. Neel, J.H. Reed, Performance evaluation of cognitive radios: metrics, utility functions, and methodology, Proc. IEEE 97 (4) (2009) 642-659. April.

[4] FCC, In the matter of unlicensed operation in the TV broadcast bands: additional spectrum for unlicensed devices below $900 \mathrm{MHz}$ and in the $3 \mathrm{GHz}$ band, September 2010, Second Memorandum Opinion and Order. <http://transition.fcc.gov/DailyReleases/ DailyBusiness/2010/db0923/FCC-10-174A1.pdf>.

[5] D. Hu, S. Mao, Design and analysis of a sensing error-aware mac protocol for cognitive radio networks, in: Proc. IEEE GLOBECOM'09, Honolulu, HI, December 2009, pp. 1-6.

[6] D. Hu, S. Mao, Resource allocation for medium grain scalable videos over femtocell cognitive radio networks, in: Proc. IEEE ICDCS'11, Minneapolis, MN, June 2011, pp. 258-267.

[7] H.-C. Lee, D.-C. Oh, Y.-H. Lee, Mitigation of inter-femtocell interference with adaptive fractional frequency reuse, in: Proc. IEEE ICC'10, Cape Town, South, Africa, May 2010, pp. 1-5.
[8] V. Angelakis, S. Papadakis, V.A. Siris, A. Traganitis, Adjacent channel interference in 802.11a is harmful: Testbed validation of a simple quantification model, IEEE Commun. Mag. 49 (3) (2011) 160-166. March.

[9] S. Kompella, S. Mao, Y.T. Hou, H.D. Sherali, On path selection and rate allocation for video in wireless mesh networks, IEEE/ACM Trans. Netw. 17 (1) (2009) 212-224. February.

[10] H. Su, X. Zhang, Cross-layer based opportunistic mac protocols for qos provisionings over cognitive radio wireless networks, IEEE J. Sel. Areas Commun. 26 (1) (2008) 118-129. January.

[11] Y.T. Hou, Y. Shi, H.D. Sherali, Spectrum sharing for multi-hop networking with cognitive radios, IEEE J. Sel. Areas Commun. 26 (1) (2008) 146-155. January.

[12] D. Hu, S. Mao, Y.T. Hou, J.H. Reed, Scalable video multicast in cognitive radio networks, IEEE J. Sel. Areas Commun. 28 (3) (2010) 334-344. April.

[13] M.S. Bazaraa, J.J. Jarvis, H.D. Sherali, Linear Programming and Network Flows, 4th ed., John Wiley \& Sons, Inc., New York, NY, 2010.

[14] A. Motamedi, A. Bahai, MAC protocol design for spectrum-agile wireless networks: Stochastic control approach, in: Proc. IEEE DySPAN'07, Dublin, Ireland, April 2007, pp. 448-451.

[15] Q. Zhao, S. Geirhofer, L. Tong, B.M. Sadler, Opportunistic spectrum access via periodic channel sensing, IEEE Trans. Signal Process. 56 (2) (2008) 785-796. February.

[16] Y. Chen, Q. Zhao, A. Swami, Joint design and separation principle for opportunistic spectrum access in the presence of sensing errors, IEEE Trans. Inf. Theory 54 (5) (2008) 2053-2071. May.

[17] R. Urgaonkar, M.J. Neely, Opportunistic scheduling with reliability guarantees in cognitive radio networks, IEEE Trans. Mobile Comput. 8 (6) (2009) 766-777. June.

[18] C. Zhao, K. Kwak, Power/bit loading in OFDM-based cognitive networks with comprehensive interference considerations: The single-SU case, IEEE Trans. Veh. Technol. 59 (4) (2010) 1910-1922. May.

[19] L. Yang, M.-S. Alouini, Performance comparison of different selection combining algorithms in presence of co-channel interference, IEEE Trans. Veh. Technol. 55 (2) (2006) 559-571. March.

[20] J. Nachtigall, A. Zubow, J.-P. Redlich, The impact of adjacent channel interference in multi-radio systems using IEEE 802.11, in: Proc. IWCMC'08, Crete Island, Greece, August 2008, pp. 874-881.

[21] M.G. Sanchez, M.A. Acuna, I. Cuinas, R.M. Rodriguez-Osorio, L. de Haro, A. Garcia-Pino, Cochannel and adjacent channel interference in actual terrestrial TV scenarios - part I: Field measurements, IEEE Trans. Broadcast. 48 (2) (2002) 111-115. June.

[22] Z.A. Shamsan, L.F. Abdulrazak, T.A. Rahman, Co-channel and adjacent channel interference evaluation for IMT-Advanced coexistence with existing fixed systems, in: Proc. IEEE International RF and Microwave Conference 2008, Kuala Lumpur, Malaysia, December 2008, pp. 6569.

[23] C.-S. Sum, R. Funada, J. Wang, T. Baykas, M.A. Rahman, H. Harada, Error performance and throughput evaluation of a multi-Gbps millimeter-wave WPAN system in the presence of adjacent and cochannel interference, IEEE J. Sel. Areas Commun. 27 (8) (2009) 14331442. October.

[24] K. Gulati, B. Evans, J. Andrews, K. Tinsley, Statistics of co-channel interference in a field of Poisson and Poisson-Poisson clustered interferers, IEEE Trans. Signal Process. 58 (12) (2010) 6207-6222. December.

[25] E. Obregon, L. Shi, J. Ferrer, J. Zander, A model for aggregate adjacent channel interference in TV white space, in: Proc. IEEE VTC-Spring 2011, Budapest, Hungary, May 2011, pp. 1-5.

[26] A.M.A. Ahmed, I. Marsland, Co-channel interference cancellation in multihop relay networks, in: Proc. IEEE ICC'08 Workshops, Beijing, P.R. China, May 2008, pp. 62-67.

[27] A.M.A. Ahmed, I. Marsland, Co-channel interference cancellation in wireless cellular networks, in: Proc. IEEE VTC-Spring 2008, Marina Bay, Singapore, May 2008, pp. 698-702.

[28] G. Miao, Y. Li, N. Himayat, S. Talwar, Co-channel interference avoidance MAC in wireless cellular networks, IEEE Trans. Commun. 57 (11) (2009) 3405-3897. November.

[29] O. Bendov, C.B. Patel, Television receiver optimization in the presence of adjacent channel interference, IEEE Trans. Broadcast. 51 (1) (2005) 38-42. March.

[30] D. Gidony, I. Kalet, Adjacent channel interference cancellation for MSK-type signals using antenna diversity in Rayleigh fading environment, IEEE Trans. Commun. 52 (2) (2004) 317-325. February. 


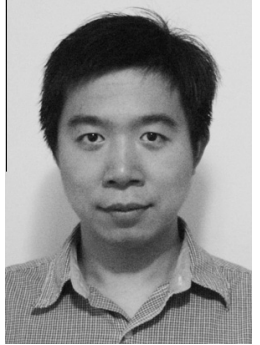

Donglin Hu received the M.S. degree from Tsinghua University, Beijing, China, in 2007 and the B.S. degree from Nanjing University of Posts and Telecommunications, Nanjing, China in 2004, respectively, all in electrical engineering. He received the M.S. degree in Probability and Statistics from Auburn University, Auburn, AL, in 2011, and the Ph.D. degree in Electrical and Computer Engineering from Auburn University in 2012. Currently he is a postdoctoral research fellow in the Department of Electrical and Computer Engineering at Auburn University. His research interests include cognitive radio networks, femtocell networks, network modeling, cross-layer design, performance analysis, and algorithm optimization for wireless networks and multimedia communications.

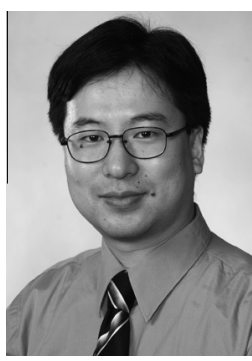

Shiwen Mao received Ph.D. in electrical and computer engineering from Polytechnic University, Brooklyn, NY (now Polytechnic Institute of New York University) in 2004. He was a research staff member with IBM China Research Lab from 1997 to 1998 . He was a Postdoctoral Research Fellow/Research Scientist in the Bradley Department of Electrical and Computer Engineering at Virginia Tech, Blacksburg, VA, USA from 2003 to 2006. Currently, he is the McWane Associate Professor in the Department of Electrical and Computer Engineering, Auburn University, Auburn, AL.
His research interests include cross-layer optimization of wireless networks and multimedia communications, with current focus on cognitive radios, femtocell networks, $60 \mathrm{GHz}$ mmWave networks, and smart grid. He is on the Editorial Board of IEEE Transactions on Wireless Communications, IEEE Communications Surveys and Tutorials, Elsevier Ad Hoc Networks Journal, Wiley International Journal of Communication Systems, and ICST Transactions on Mobile Communications and Applications. He serves as the Director of E-Letter of IEEE Communications Society's Multimedia Communications Technical Committee (MMTC), for 2012-2014.

He is a coauthor of TCP/IP Essentials: A Lab-Based Approach (Cambridge University Press, 2004). He was awarded the McWane Endowed Professorship in the Samuel Ginn College of Engineering for the Department of Electrical and Computer Engineering, Auburn University in August 2012. He received the US National Science Foundation Faculty Early Career Development Award (CAREER) in 2010. He is a co-recipient of The 2004 IEEE Communications Society Leonard G. Abraham Prize in the Field of Communications Systems and The Best Paper Runner-up Award at The Fifth International Conference on Heterogeneous Networking for Quality, Reliability, Security and Robustness (QShine) in 2008. He also received Auburn Alumni Council Research Awards for Excellence-Junior Award in 2011 and two Auburn Author Awards in 2011. He holds one US patent. 